European

Thyroid Journal
Eur Thyroid J 2014;3:179-188

DOI: $10.1159 / 000365145$
Received: May 20, 2014

Accepted after revision: June 6, 2014 Published online: August 29, 2014

\title{
Challenges in the Evaluation of Urinary lodine Status in Pregnancy: The Importance of lodine Supplement Intake and Time of Sampling
}

\author{
Stine Linding Andersen ${ }^{a, c}$ Louise Kolding Sørensen ${ }^{a} \quad$ Anne Krejbjerg ${ }^{a, c}$ \\ Margrethe Møller ${ }^{b}$ Peter Laurberg ${ }^{\mathrm{a}, \mathrm{c}}$ \\ Departments of ${ }^{\mathrm{a}}$ Endocrinology and ${ }^{\mathrm{b}}$ Obstetrics and Gynecology, Aalborg University Hospital, and \\ 'Department of Clinical Medicine, Aalborg University, Aalborg, Denmark
}

\section{Key Words \\ Urinary iodine concentration - Urinary creatinine concentration $\cdot 24$-Hour urinary iodine excretion . \\ Pregnancy $\cdot$ Household $\cdot$ lodine supplement}

\begin{abstract}
Objectives: Median urinary iodine concentration (UIC) is the recommended method to evaluate iodine status in pregnancy, but several factors may challenge the interpretation of the results. We evaluated UIC in pregnant women according to (1) sampling in the hospital versus at home, (2) time of the most recent iodine supplement intake prior to sampling, and (3) members of their household. Study Design: Danish crosssectional study in the year 2012. Pregnant women $(n=158)$, their male partners $(n=157)$ and children $(n=51)$ provided a questionnaire with detailed information on iodine supplement intake and a spot urine sample obtained in the hospital and/or at home for measurement of UIC and urinary creatinine concentration. Results: In the pregnant women providing a urine sample both in the hospital and at home $(n=66)$, individual UIC ( $p=0.002)$ and urinary creatinine concentration ( $p=0.042$ ), but not estimated 24-hour urinary iodine excretion ( $p=0.79$ ), were higher when sampling was at home. Median UIC was dependent on the time of the most
\end{abstract}

recent iodine supplement intake prior to sampling [same day ( $\mathrm{n}=79): 150 \mu \mathrm{g} / \mathrm{l}(95 \% \mathrm{Cl} 131-181 \mu \mathrm{g} / \mathrm{l})$, the day before ( $\mathrm{n}=51): 105 \mu \mathrm{g} / \mathrm{l}(78-131 \mu \mathrm{g} / \mathrm{l})$, several days ago/non-user ( $\mathrm{n}=28): 70 \mu \mathrm{g} / \mathrm{l}(56-94 \mu \mathrm{g} / \mathrm{l}), \mathrm{p}<0.001]$. The pattern was similar in the male partners. Apart from a more frequent iodine supplement intake in pregnancy $(87.3 \%$ vs. partners $15.9 \%)$, no systematic differences were observed in urinary measurements between the pregnant women and their partners. Conclusions: Time of spot urine sampling and time span from iodine supplement intake to spot urine sampling should be considered when evaluating urinary iodine status in pregnancy.

(c) 2014 European Thyroid Association Published by S. Karger AG, Basel

\section{Introduction}

Population median urinary iodine concentration (UIC) is the recommended method to assess iodine status [1]. UIC in pregnancy is extensively studied and adequate maternal iodine intake is of major concern [2-4]. Guidelines recommend the use of spot urine samples and the estimation of median UIC in a population of pregnant women, and results are compared to the recommended 
Fig. 1. Flowchart illustrating the selection of participants. Exclusions in the group 'participating in the hospital only' were: no urine sample from the pregnant woman $(\mathrm{n}=1)$ and not a male partner $(\mathrm{n}=1)$. Exclusions in the group 'participating in the hospital and at home' were: pregnant woman with gastric bypass $(\mathrm{n}=1)$, pregnant women with inflammatory bowel disease $(\mathrm{n}=1)$, pregnant woman with inconsistent information on iodine supplement intake between samplings $(n=1)$, partner did not complete the questionnaire $(\mathrm{n}=2)$, and not a male partner $(\mathrm{n}=2)$.

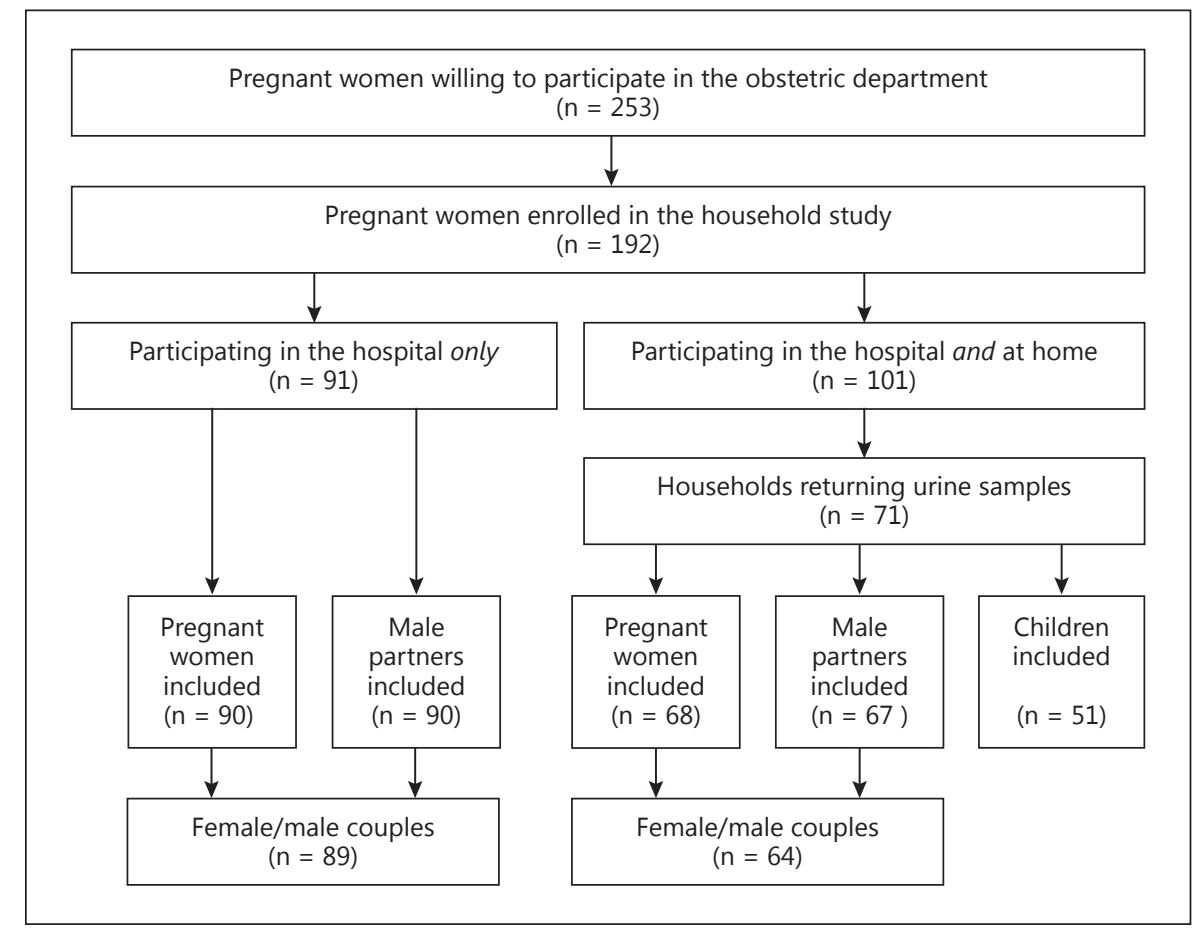

level during pregnancy $(150-249 \mu \mathrm{g} / \mathrm{l})$ defined by the World Health Organization (WHO), the United Nation Children's Fund (UNICEF) and the International Council for the Control of Iodine Deficiency Disorders (ICCIDD) [1]. Another method to study iodine intake is to measure iodine excretion in a full 24-hour urine collection or to estimate 24-hour iodine excretion based on measurement of both iodine and creatinine concentration in a spot urine sample [5].

Physiological changes occur in both thyroid and renal function during pregnancy which may challenge the interpretation of the results [6]. We previously reported that iodine supplement intake in Danish pregnant women was frequent, but median UIC was low and below the level recommended in pregnancy both in iodine supplement users and non-users, and also when UIC was adjusted by urinary creatinine to estimate 24-hour urinary iodine excretion [7].

In our study [7] and in the majority of studies evaluating iodine status in pregnant women, urine samples are obtained during a routine antenatal hospital visit. We speculated whether urine samples obtained at hospital were representative of daily life in pregnant women. Thus, we expanded our previous study to include both a urine sample obtained during the hospital visit for routine antenatal ultrasound in pregnancy and a urine sample ob- tained a subsequent day, when the pregnant woman was at home.

It has been reported that the median UIC in family members such as schoolchildren are not representative for UIC in pregnant women [8]. We speculated whether at least some of this difference might be caused by the pregnant women and their family member being investigated under different conditions. To evaluate this hypothesis, we sampled urine for iodine measurements both from the pregnant women and from members of their household under identical conditions.

In our previous study [7], the majority of pregnant women took iodine supplements. Finally, we speculated whether the timing of the most recent iodine supplement intake prior to spot urine sampling would have an influence on the UIC obtained and thus the results of iodine status evaluation.

\section{Materials and Methods}

Study Population and Design

The pregnant women enrolled in the present study took part in our investigation on iodine intake during pregnancy in an area of Denmark with previously moderate iodine deficiency [7]. From June 13 to August 10, 2012, we consecutively recruited healthy, pregnant women referred to Aalborg University Hospital for ob- 
Table 1. Characteristics of the pregnant women and their household members at the time of inclusion in the study

\begin{tabular}{|c|c|c|c|}
\hline & $\begin{array}{l}\text { Pregnant women } \\
(\mathrm{n}=158)\end{array}$ & $\begin{array}{l}\text { Partners } \\
(\mathrm{n}=157)\end{array}$ & $\begin{array}{l}\text { Children } \\
(\mathrm{n}=51)\end{array}$ \\
\hline Male gender & NA & $157(100.0)$ & $26(51.0)$ \\
\hline Danish ethnicity ${ }^{\mathrm{a}}$ & $151(95.6)$ & $149(94.9)$ & $49(96.1)$ \\
\hline Age, years & $30(19-41)$ & $32(23-49)$ & $6(1-14)$ \\
\hline Weight, kg ${ }^{\mathrm{b}}$ & $67(48-114)$ & $85(56-135)$ & $19(9-70)$ \\
\hline Height, $\mathrm{cm}^{\mathrm{c}}$ & $169(151-182)$ & $183(166-201)$ & $115(74-169)$ \\
\hline $\mathrm{BMI}^{\mathrm{d}}$ & $23.4(17.6-41.0)$ & $25.3(19.2-39.9)$ & $15.5(12.7-29.1)$ \\
\hline$<25$ & $93(60.0)$ & $70(45.2)$ & $45(97.8)$ \\
\hline $25-29.9$ & $36(23.2)$ & $69(44.5)$ & $1(2.2)$ \\
\hline$\geq 30$ & $26(16.8)$ & $16(10.3)$ & $0(0)$ \\
\hline \multicolumn{4}{|l|}{ Smoking ${ }^{\mathrm{e}}$} \\
\hline Current & $6(3.8)$ & $27(17.3)$ & NA \\
\hline Previous & $52(32.9)$ & $33(21.2)$ & NA \\
\hline Never & $100(63.3)$ & $96(61.5)$ & NA \\
\hline \multicolumn{4}{|l|}{ Educational level $^{\mathrm{f}}$} \\
\hline Basic & $11(7.0)$ & $26(16.6)$ & NA \\
\hline Low & $21(13.3)$ & $43(27.4)$ & NA \\
\hline Middle & $81(51.3)$ & 47 (29.9) & NA \\
\hline High & $45(28.4)$ & $41(26.1)$ & NA \\
\hline Supplement not containing iodine & $13(8.2)$ & $15(9.6)$ & $2(3.9)$ \\
\hline Iodine supplement & $138(87.3)$ & $25(15.9)$ & $13(25.5)$ \\
\hline $175 \mu \mathrm{g} /$ day & 111 & 0 & 0 \\
\hline $150 \mu \mathrm{g} /$ day & 24 & 22 & 0 \\
\hline $70 \mu \mathrm{g} /$ day & 0 & 0 & 12 \\
\hline Others ${ }^{g}$ & 3 & 3 & 1 \\
\hline
\end{tabular}

Values are $\mathrm{n}, \mathrm{n}(\%)$ or median (range). ${ }^{\mathrm{a}}$ Two children had 1 parent of Danish origin and 1 parent of non-Danish origin. ${ }^{b}$ Values are pre-pregnancy weight for the pregnant women. Missing values on weight $(n=7)$ not included. ${ }^{c}$ Missing values on height $(n=6)$ not included. ${ }^{d}$ Missing values on BMI $(n=9)$ not included. Pre-pregnancy BMI for the pregnant women. ${ }^{\mathrm{e}}$ Missing value on smoking $(\mathrm{n}=1)$ not included. ${ }^{\mathrm{f}}$ Highest educational level achieved or initiated. 'Basic' (primary/secondary education only; 9-13 years), 'low' (vocational education and training: 9-13 years), 'middle' (short or medium cycle higher education: 14-16 years), 'high' (long cycle higher education: $\geq 17$ years). ${ }^{g}$ Pregnant women: $87.5 \mu \mathrm{g} /$ day $(\mathrm{n}=2), 350 \mu \mathrm{g} /$ day $(\mathrm{n}=1)$; partners: $300 \mu \mathrm{g} /$ week $(\mathrm{n}=1), 300 \mu \mathrm{g} / \mathrm{day}(\mathrm{n}=$ 2); children: $75 \mu \mathrm{g} /$ day $(\mathrm{n}=1)$.

stetric ultrasound as part of the antenatal investigation program (fig. 1). After informed consent, the women were asked to fill out a questionnaire, to list detailed information on dietary supplements and to deliver a spot urine sample.

Members of their household were recruited in one of the following ways: (1) the partner filled out a questionnaire and delivered a spot urine sample in the hospital at the same time as the pregnant woman or (2) only the pregnant woman participated in the hospital and questionnaires and vials for urine sampling were handed out to the pregnant woman and her household members for sampling at home (fig. 1). Thus, 66 pregnant women participating with the household at home delivered a urine sample both at the time of inclusion in the hospital and at home (2 women only delivered a urine sample at home). Participants were instructed to perform non-fasting urine sampling as close in time as possible for all household members and to list information on iodine supple- ment intake including the time of the most recent supplement intake prior to urine sampling.

The study protocol was approved by the local ethical committee.

\section{Laboratory Procedures}

Urine samples were stored at $-20^{\circ} \mathrm{C}$ until measurement of UIC in runs during the time of study inclusion. UIC was determined by the cerium/arsenite method after alkaline ashing, as previously described [9]. The analytical sensitivity was $2 \mu \mathrm{g} / \mathrm{l}$ and the recovery of iodine was 95.5\% (SEM 2.4\%). When a urine sample (UIC 93.9 $\mu \mathrm{g} / \mathrm{l})$ was measured in triplicates in 18 assays, the intra-assay CV was $2.1 \%$ and the inter-assay CV was $2.7 \%$ for single determination [10]. The iodine laboratory was certified by the US Centers for Disease Control and Prevention EQUIP program. Urinary creatinine concentrations were measured on a Cobas 8000 system (Roche, 
Table 2. UIC, urinary creatinine concentration and estimated 24-hour iodine excretion in Danish pregnant women and their household members

\begin{tabular}{|c|c|c|c|c|c|c|c|c|c|c|}
\hline & \multicolumn{3}{|c|}{ All participants } & \multicolumn{3}{|c|}{ Iodine supplement } & \multicolumn{3}{|c|}{ No iodine supplement } & $\mathrm{p}^{\mathrm{a}}$ \\
\hline \multicolumn{11}{|l|}{$U I C, \mu g / l$} \\
\hline Pregnant women & 158 & 119 & $67-180$ & 138 & 130 & $69-203$ & 20 & 76 & $55-132$ & 0.008 \\
\hline Sampling in the hospital & 90 & 105 & $59-208$ & 79 & 112 & $65-213$ & 11 & 59 & $21-93$ & 0.021 \\
\hline Sampling at home & 68 & 134 & $85-177$ & 59 & 136 & $93-180$ & 9 & 98 & $62-132$ & 0.10 \\
\hline Sampling at home & 67 & 115 & $80-150$ & 10 & 136 & $91-175$ & 57 & 110 & $77-137$ & 0.23 \\
\hline Children & 51 & 126 & $102-157$ & 13 & 151 & $116-202$ & 38 & 121 & $98-150$ & 0.035 \\
\hline \multicolumn{11}{|c|}{ Urinary creatinine concentration, $\mathrm{mmol} / \mathrm{l}^{\mathrm{b}}$} \\
\hline Pregnant women & 158 & 7.6 & $4.2-12.6$ & 138 & 7.2 & $3.9-11.8$ & 20 & 11.8 & $5.7-14.2$ & 0.051 \\
\hline Sampling in the hospital & 90 & 6.4 & $3.4-11.1$ & 79 & 5.9 & $3.4-10.6$ & 11 & 9.1 & $3.6-14.3$ & 0.30 \\
\hline Children & 51 & 7.2 & $4.8-10.3$ & 13 & 6.3 & $4.7-7.8$ & 38 & 7.4 & $5.1-11.6$ & 0.28 \\
\hline \multicolumn{11}{|c|}{ Estimated 24-hour iodine excretion, $\mu \mathrm{g} / 24 \mathrm{~h}$} \\
\hline Pregnant women ${ }^{c}$ & 158 & 164 & $109-263$ & 138 & 174 & $123-278$ & 20 & 86 & $56-100$ & $<0.001$ \\
\hline Sampling in the hospital & 90 & 164 & $114-309$ & 79 & 172 & $121-346$ & 11 & 83 & $54-109$ & $<0.001$ \\
\hline Sampling at home & 68 & 159 & $105-211$ & 60 & 175 & $123-228$ & 9 & 87 & $77-96$ & $<0.001$ \\
\hline Male partners ${ }^{\mathrm{d}}$ & 157 & 113 & $85-145$ & 25 & 136 & $102-217$ & 132 & 110 & $82-137$ & 0.013 \\
\hline Sampling in the hospital & 90 & 105 & $82-136$ & 15 & 136 & $102-323$ & 75 & 103 & $81-125$ & 0.020 \\
\hline Sampling at home & 67 & 122 & $92-160$ & 10 & 137 & $94-196$ & 57 & 122 & $86-145$ & 0.31 \\
\hline Children $^{\mathrm{e}}$ & 51 & 63 & $49-101$ & 13 & 66 & $59-103$ & 38 & 63 & $48-82$ & 0.19 \\
\hline
\end{tabular}

Rotkreuz, Switzerland). Equipment was calibrated according to the manufacturer's instructions and external standards were included.

\section{Statistical Analyses}

Urinary iodine excretion was expressed as spot urine concentration ( $\mu \mathrm{g}$ iodine $/ \mathrm{l}$ ) and as estimated 24 -hour iodine excretion ( $\mu \mathrm{g}$ iodine/24 h) calculated from the reported mean 24-hour urinary creatinine excretion in a group of Danish pregnant women $(1.09 \mathrm{~g}$ creatinine/24 h) [11], Belgian men aged 25-49 years (1.74 g creatinine/24 h) [12], and German children [13] (mean values by gender and height; missing height $(\mathrm{n}=4)$ was substituted by the average height for age in Danish children [14]).

UIC showed skewed distribution, and results were expressed as medians with 25 th and 75 th percentiles (interquartile range, IQR) or $95 \%$ confidence interval $(95 \% \mathrm{CI})$. The Mann-Whitney U test was used to compare median urinary measurements by independent groups (iodine supplement vs. no iodine supplement and hospital vs. at home), and the Kruskal-Wallis test was used for comparison by time of the most recent iodine supplement intake. For paired analyses (two samples from the same pregnant woman and pregnant woman vs. male partner), the Wilcoxon signed-rank test was applied. For linear regression, logarithmically transformed urinary measurements were used.

Statistical analyses were performed using Stata 11 (StataCorp, College Station, Tex., USA) and a 5\% level of significance was chosen.

\section{Results}

\section{Study Population}

Among the pregnant women enrolled in the household study (fig. 1), the median gestational week at the initial inclusion in the hospital was week 20 (range 11-37), 


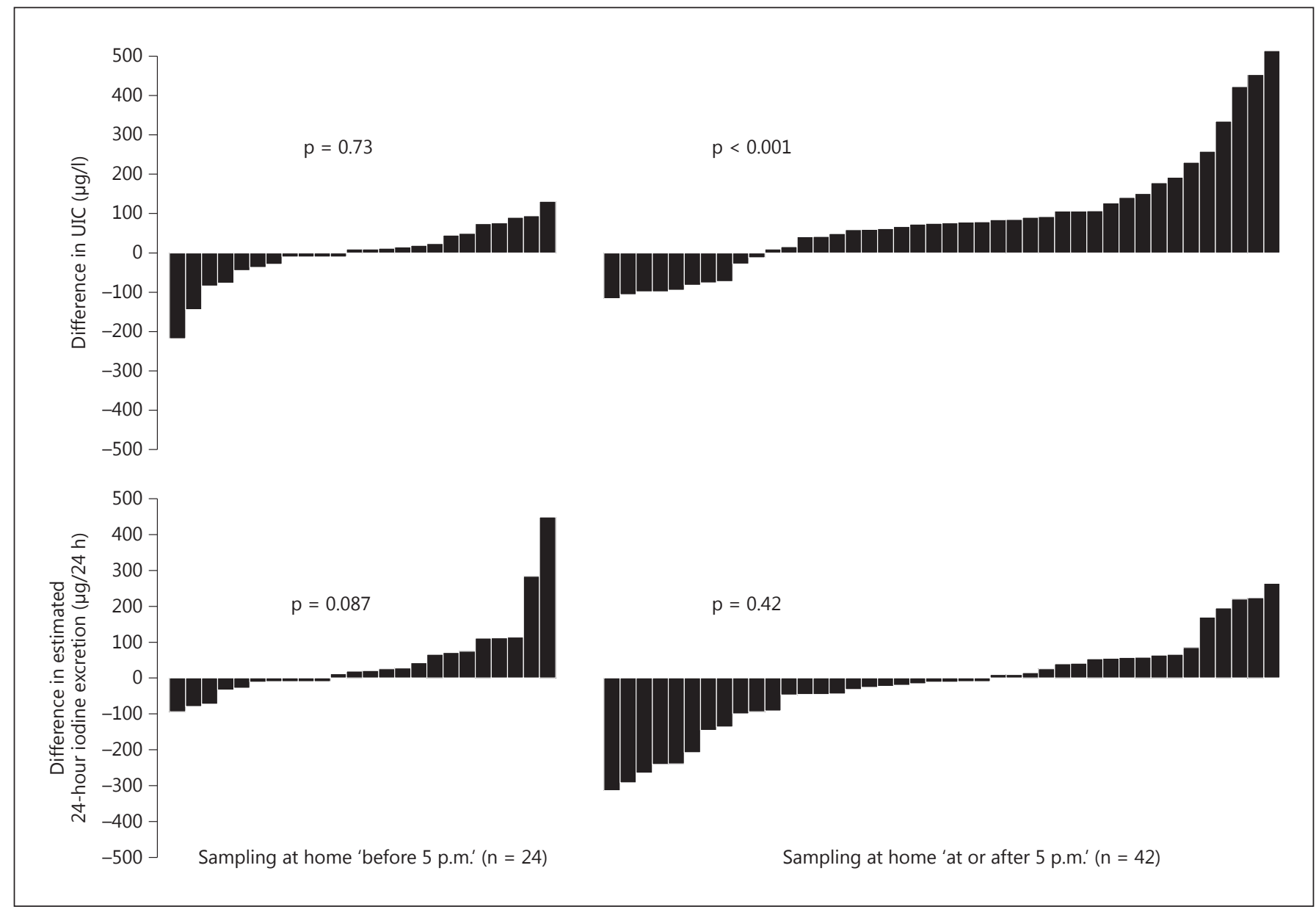

Fig. 2. Bar charts illustrating the value at home minus the value in the hospital in UIC (upper row) and estimated 24-hour urinary iodine excretion (lower row) for the 66 pregnant women who delivered a spot urine sample both in the hospital at inclusion and later at home. Results are stratified according to the time of sam- pling at home: left column was before 5 p.m. and right column was at or after 5 p.m. Differences in the range from -1 to $-9(n=6)$ were set to -10 and differences in the range from 0 to $9(n=5)$ were set to 10 for illustration. $\mathrm{p}$ values are results of the Wilcoxon signed-rank test: at home versus in the hospital. and $62 \%$ were expecting their first child. Table 1 describes characteristics of the pregnant women and their household members. Current smoking was more frequent among the male partners, and also the frequency of dietary supplement intake differed. Intake of iodine supplement in pregnancy was common, and the iodine supplements most frequently used contained $175 \mu \mathrm{g}$ iodine/day. On the other hand, iodine supplement intake was less common among both male partners and children, and the iodine supplements used contained less iodine.

\section{Urinary Measurements}

Overall, the median UIC among adults (pregnant women and male partners, $\mathrm{n}=315)$ was $104 \mu \mathrm{g} / \mathrm{l}(\mathrm{IQR}$
61-150) and the estimated 24-hour iodine excretion was $129 \mu \mathrm{g} / 24 \mathrm{~h}$ (IQR 95-193). In general, both median UIC and estimated 24-hour urinary iodine excretion were higher when iodine supplement was used (table 2).

\section{Urine Sampling in the Hospital versus at Home}

In the pregnant women and in the male partners, both UIC and urinary creatinine concentration (table 2) tended to be higher at home than in the hospital (at home vs. hospital: median UIC all pregnant women $\mathrm{p}=0.17$, all male partners $\mathrm{p}<0.001$; median urinary creatinine concentration: all pregnant women $\mathrm{p}=0.060$, all male partners $\mathrm{p}=0.006)$. On the other hand, when urinary creatinine concentration was used to estimated 24 -hour uri- 


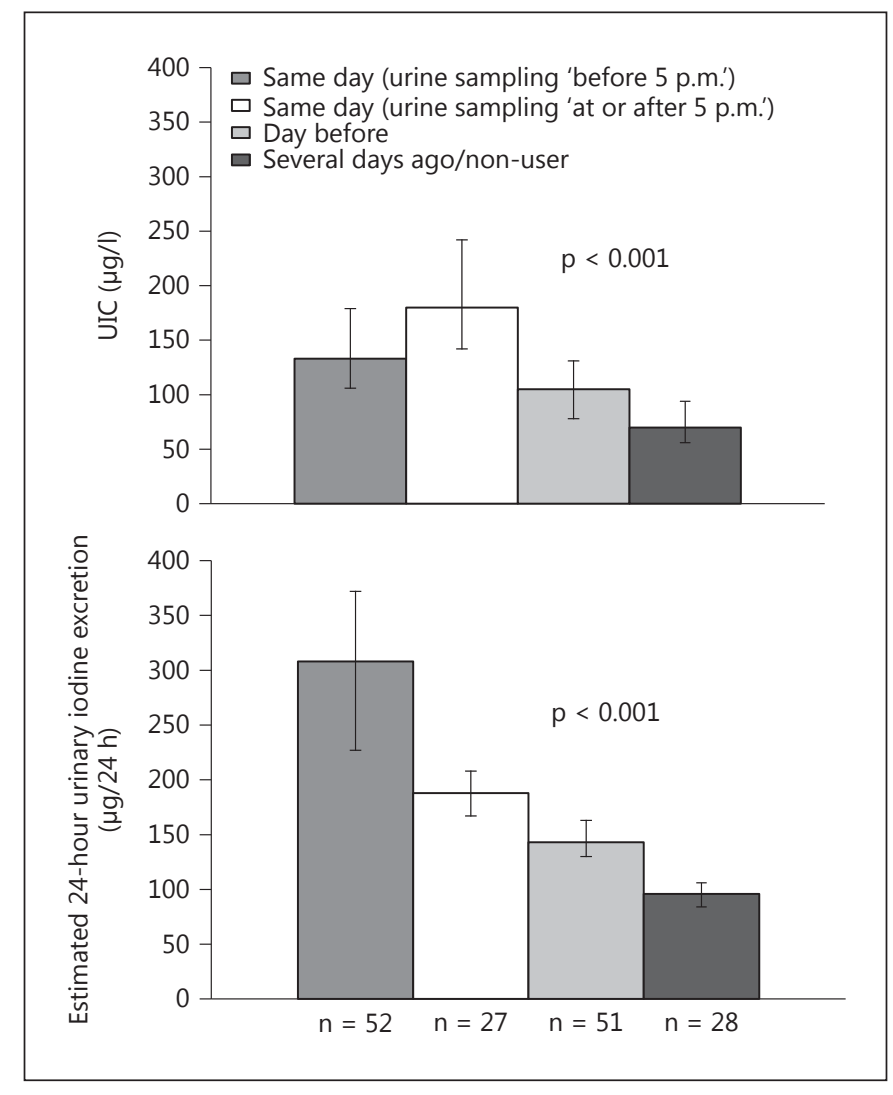

Fig. 3. Median (95\% CI) UIC (upper figure) and estimated 24-hour urinary iodine excretion (lower figure) in 158 pregnant women stratified by time of the most recent iodine supplement intake prior to urine sampling. $p$ values are results of the Kruskal-Wallis test: the same day with sampling before 5 p.m. versus the same day with sampling at or after 5 p.m. versus the day before versus several days ago/non-user.

naryiodine excretion, the differences in results diminished (all pregnant women $\mathrm{p}=0.25$, all male partners $\mathrm{p}=0.10$ ).

Individual comparison in the 66 pregnant women who delivered a urine sample both in the hospital and at home also showed that UIC and urinary creatinine concentration were higher at home (median UIC $133 \mu \mathrm{g} / \mathrm{l} \mathrm{vs.}$ in the hospital $84 \mu \mathrm{g} / \mathrm{l}, \mathrm{p}=0.002$; median urinary creatinine concentration 9.2 vs. $5.7 \mathrm{mmol} / \mathrm{l}$ in the hospital, $\mathrm{p}=$ 0.042 ), whereas no difference in estimated 24-hour urinary iodine excretion was observed (159 vs. $154 \mu \mathrm{g} / 24 \mathrm{~h}$ at home, $\mathrm{p}=0.79$ ).

In a post hoc analysis, we evaluated whether the time of urine sampling could be involved in our findings. All children delivered the urine sample at home, and their time of sampling was at median 5 p.m. (IQR 4-6 p.m.). The pregnant women and male partners participated either in the hospital or at home, and the time of sampling differed between the two locations: hospital: median 10 a.m. (IQR 9-11 a.m.), home: median 6 p.m. (IQR 3-8 p.m.). Thus, the majority of families had performed the sampling at home in the afternoon or evening, whereas sampling in the hospital was in the morning.

We focused our post hoc analysis on the 66 pregnant women who delivered a urine sample both in the hospital and at home. Differences in UIC and estimated 24-hour iodine excretion in the 66 pregnant women who delivered a urine sample both in the hospital and at home are depicted in figure 2 . The women were stratified according to the time of sampling at home: 'before 5 p.m.' (median 2 p.m., IQR 10 a.m. to 4 p.m.) and 'at or after 5 p.m.' (median 7 p.m., IQR 6-9 p.m.). No difference was observed in UIC and estimated 24-hour iodine excretion between hospital and home samples when sampling at home was performed 'before 5 p.m.'. On the other hand, when sampling at home was performed 'at or after 5 p.m.', UIC was significantly higher at home, whereas no difference was observed in estimated 24-hour iodine excretion.

Pregnant Women versus Members of Their Household We performed a pairwise comparison of UIC in female/male couples stratified according to iodine supplement intake. In the largest group, where only the pregnant woman took iodine supplement ( $\mathrm{n}=109$ couples), UIC was significantly higher in the pregnant woman than in the male partner as might be expected (median UIC 130 vs. $91 \mu \mathrm{g} / \mathrm{l}$ in the male partners, $\mathrm{p}<0.001)$. On the other hand, no significant difference in UIC between the pregnant woman and her male partner was observed, when both ( $\mathrm{n}=24$ couples; median UIC $122 \mathrm{vs.} 91 \mu \mathrm{g} / \mathrm{l}$ in the male partners, $\mathrm{p}=0.90)$ or none ( $\mathrm{n}=17$ couples; median UIC 70 vs. $95 \mu \mathrm{g} / \mathrm{l}$ in the male partners, $\mathrm{p}=0.20$ ) took iodine supplement, with similar results for estimated 24hour urinary iodine excretion (data not shown).

Median UIC in the children (table 2) was not significantly different from the median UIC in the pregnant women $(p=0.89)$ and in the male partners $(p=0.074)$ sampling at home and results were similar when stratified by iodine supplement intake (data not shown). In multivariate linear regression including age of the child (grouped by quartiles), gender of the child (male/female) and iodine supplement intake (yes/no), higher age was a significant predictor of higher urinary creatinine concentration ( $\mathrm{p}<0.001)$, but not UIC $(\mathrm{p}=0.45)$ and 24-hour estimated iodine excretion $(\mathrm{p}=0.66)$. Gender of the child was not a significant predictor of UIC $(\mathrm{p}=0.55)$.

Altogether 29 families delivered a urine sample from the pregnant woman, the male partner and 1-3 children. 
Multivariate linear regression including iodine supplement intake and type of family member did not indicate that UIC was significantly different between family members [pregnant woman (reference), male partner $(\mathrm{p}=$ $0.81)$, child $(\mathrm{p}=0.50)]$.

\section{Time from Iodine Supplement Intake to Urine Sampling}

Both UIC and estimated 24-hour iodine excretion were influenced by the timing of the most recent iodine supplement intake prior to urine sampling (fig. 3). Median values were higher when iodine supplement intake was the same day prior to urine sampling (first and second bar). Looking only at groups with iodine supplement intake the same day as urine sampling (first and second bar), results were different for UIC and estimated 24hour iodine excretion (fig. 3). Considering this in relation to figure 2, UIC was influenced by both the time of sampling and the time of the most recent iodine supplement intake, whereas mainly the time of the most recent iodine supplement intake influenced estimated 24-hour iodine excretion.

Among the male partners, the trend was similar to that observed in pregnancy [iodine supplement intake the same day $(\mathrm{n}=10)$ : median UIC $152 \mu \mathrm{g} / \mathrm{l}$ (95\% CI 94-296 $\mu \mathrm{g} / \mathrm{l})$, the day before $(\mathrm{n}=11): 124 \mu \mathrm{g} / \mathrm{l}(34-175 \mu \mathrm{g} / \mathrm{l})$, several days ago/non-user $(\mathrm{n}=134): 90 \mu \mathrm{g} / \mathrm{l}(77-101 \mu \mathrm{g} / \mathrm{l})$, $\mathrm{p}=0.004]$.

\section{Sensitivity Analyses}

For the 66 pregnant women who delivered two urine samples, the interval between the two samples was median 16 days (IQR 6-29). When analyses were stratified by the median time between samplings, results were the same as depicted in figure 2 (data not shown). None of the 66 women had made any change in iodine supplement intake between the two samples, and 57 women took iodine supplement. Considering the time of the most recent iodine supplement intake prior to urine sampling, results (fig. 2) were similar when analyses were restricted to the 44 women with iodine supplement the same time prior to both the first and the second sampling (data not shown).

\section{Discussion}

\section{Principle Findings}

This study evaluates urinary iodine status in Danish pregnant women according to the location and time of spot urine sampling, the time of the most recent iodine supplement intake prior to spot urine sampling and in comparison to members of the household. Differences in urinary measurements between the hospital and at home sampling were largely explained by differences in time of sampling during the day. UIC and urinary creatinine concentration were higher when sampling was performed in the evening, but when UIC was adjusted by urinary creatinine, the time-dependent differences leveled out. The time of the most recent iodine supplement intake prior to sampling was a predictor of UIC and in general, no systematic differences were observed between the pregnant woman and her male partner except that iodine supplement use was much more frequent in pregnancy.

\section{Iodine Metabolism in the Pregnant versus the \\ Non-Pregnant State}

Inorganic iodine is almost completely absorbed from the gastrointestinal tract [15], and plasma inorganic iodine is primarily balanced by iodine intake, transfer of iodine into the thyroid gland and renal excretion of iodine. UIC reflects iodine intake as $>90 \%$ of ingested iodine is excreted into the urine [1]. Physiologically, the pregnant state differs from the non-pregnant state by: iodine transport to the fetus across the placenta [16], increased use of iodine in the thyroid gland [17], and increased renal excretion of iodine (the glomerular filtration is $50 \%$ higher by the end of first trimester which is maintained throughout pregnancy [18]). Following this, the recommended range for median UIC in a population of pregnant women is $150-249 \mu \mathrm{g} / \mathrm{l}$, whereas in the nonpregnant state, median UIC in the range from 100 to 199 $\mu \mathrm{g} / \mathrm{l}$ indicates adequate iodine intake [1].

In many populations, the use of iodine supplements in pregnancy is important to ensure adequate iodine intake $[7,19,20]$, and it is of particular importance in women with a low intake of iodine-containing foods. Severe iodine deficiency in pregnancy can cause fetal brain damage [1], but recent studies have shown that also less severe iodine deficiency in pregnant women may associate with lower cognitive scores in the child $[3,4]$.

\section{UIC and Time of Sampling}

Controversies in urinary iodine determinations have been discussed for years [21]. One aspect is the time of sampling during the day. In a Danish study of 22 individuals [22], estimated 24-hour iodine excretion from a fasting morning spot urine sample was significantly lower than the actual iodine excretion measured in a 24hour sample. In a study from Switzerland [23], a total of 3,023 spot urine samples were collected from 42 indi- 
viduals, and a circadian rhythm in UIC was observed with the lowest levels in the morning and increasing levels in the afternoon and evening. UIC peaks occurred 4-5 $\mathrm{h}$ after main meals, and the time of the peaks was different for children and adults. As discussed by the authors, this may reflect different intake of iodine-containing food and a close relationship between recent iodine intake and the UIC profile. In our study, both UIC and urinary creatinine concentration were higher in samples obtained at or after 5 p.m. We speculate whether the evening meal may have influenced UIC and urinary creatinine concentration in samples obtained in the evening and/or if the urine production was smaller at that time of the day.

The 'golden standard' to measure urinary iodine excretion is to collect one or preferably several full 24-hour urine samples [1]. This would alleviate problems related to the time span between meals, supplement intake and spot urine sampling. However, a 24-hour full urine collection is often unrealistic in field studies and optimally an objective marker such as $p$-aminobenzoic acid should be used to verify the completeness [24].

\section{UIC and Recent Iodine Supplement Intake}

In a study of 16 lactating women, Leung et al. [25] showed that an acute, high intake of iodine gave rise to an increase in breast milk iodine concentration with a peak median $6 \mathrm{~h}$ after ingestion, but median UIC remained stable over the study period $(8 \mathrm{~h})$. We recently showed [26] that breast milk iodine concentration was dependent on the time of the most recent iodine supplement intake (not acute high dose) in a similar pattern to what we now illustrate for median UIC in both the pregnant women and their male partners. However, more studies are needed to corroborate results both in pregnant and non-pregnant populations.

\section{UIC in Pregnant Women versus Male Partners}

The mandatory iodine fortification of salt was introduced in Denmark in the year 2000, and the Danish investigation of iodine intake and thyroid disease (DanThyr) has monitored the iodine excretion in two regions of Denmark with a different iodine intake before the iodine fortification (moderate vs. mild iodine deficiency) [10]. The most recent data after the iodine fortification (2008-2010) have shown an overall increase in iodine excretion in both regions, but with a median UIC just below the level recommended [10]. The DanThyr study initially included women aged 18-65 years (mainly non-pregnant) and men aged 60-65 years, urine sam- ples were obtained between 8.00 a.m. and 5.30 p.m., and $35 \%$ of participants used iodine supplements [10]. We examined only pregnant women, men in younger age groups, and part of the urine samples were obtained after 5.30 p.m. Median UIC (women and men combined) was higher in our study (104 $\mu \mathrm{g} / \mathrm{l}$ vs. DanThyr $83 \mu \mathrm{g} / \mathrm{l}$ [10]), which could be explained by the higher frequency of iodine supplement use, the later time of sampling, or both. When looking at the estimated 24-hour iodine excretion $(129 \mu \mathrm{g} / 24 \mathrm{~h}$ vs. DanThyr $125 \mu \mathrm{g} / 24 \mathrm{~h}$ [10]) or at median UIC among no iodine supplement users only $(89 \mu \mathrm{g} / \mathrm{lvs}$. DanThyr $74 \mu \mathrm{g} / 1$ [27]), the difference in study results was less pronounced.

The only disparity between the pregnant women and their male partners that we observed was the more frequent use of iodine supplements in pregnancy. The couples appeared similar in terms of differences in urinary concentrations according to the time of sampling and the time of the most recent iodine supplement intake, and no significant differences were observed in the pairwise comparison of UIC when they both used or did not use iodine supplement. UIC reflects recent iodine intake, and our results indicate that the pregnant women and their male partners had comparable intake of iodine. Living in the same household implies the same drinking water and also to some extent the same diet. In our Danish study population, it did not seem as if the pregnant woman had changed her diet to a considerably different iodine intake from the male partner.

\section{UIC in Pregnant Women versus Children}

Traditionally, the median UIC in schoolchildren has been the recommended method for the assessment of iodine status in populations, including pregnant women [15]. A few studies have evaluated median UIC in schoolchildren as a proxy for iodine status in pregnancy in a household design $[8,28]$. In these studies, median UIC was higher in the children than in the pregnant women suggesting that median UIC in school-aged children should not be used for monitoring iodine status in pregnancy. In general, the median UIC in children is often higher than that of pregnant women, although it may be the other way around [29]. Notably, none of the studies reported on the time of urine sampling. In one study [8], urine from the pregnant woman was sampled in the antenatal clinic or factory, whereas the child sampled at home. Considering our results, this disparity may itself lead to differences in results when looking at UIC alone and not adjusted by urinary creatinine. 
Our study is the first study to report data on urinary iodine excretion in Danish children. The median UIC was within the level recommended in both iodine supplement users and non-users [1]. We were able to compare household members who sampled at home around the same time, and we found no significant difference in UIC when comparing children to pregnant (and non-pregnant) adults. However, the number of children was limited and an examination of schoolchildren in Denmark is needed. More studies in different populations should be performed to clarify the use of non-pregnant populations as a proxy for iodine status in pregnancy.

\section{Perspective}

This study was designed to evaluate some of the challenges in the interpretation of urinary iodine status in pregnancy. Although the number of pregnant women and household members recruited was sufficient for the evaluation of median UIC in a population [30], the disparity in use of iodine supplements among pregnant women and male partners made the numbers small in some of the stratified analyses.

The family members were instructed to perform the urine sampling at home as close in time as possible, and to facilitate this, the time of sampling was not specified except that they were instructed to make the urine samples non-fasting. Following this design, the majority of families had sampled the urine in the afternoon or evening. In our post hoc analyses it appeared that the time of sampling did influence the urinary measurements obtained, and to evaluate the possible role of time and location of sampling in more detail, it is preferable to design a study where the time of sampling is fixed and similar in both locations.

More studies are needed to corroborate results, but the findings advocate that studies evaluating iodine status in pregnant women (as well as in non-pregnant populations) from spot urine samples optimally should report the time of urine sampling and the time of the most recent iodine supplement intake prior to urine sampling and measure urinary creatinine concentration.

\section{Disclosure Statement}

The authors have no conflicts of interest to disclose.

\section{References}

1 WHO, UNICEF, ICCIDD: Assessment of Iodine Deficiency Disorders and Monitoring Their Elimination. A Guide for Programme Managers. Geneva, WHO, 2007, pp 1-99.

2 Gunnarsdottir I, Dahl L: Iodine intake in human nutrition: a systematic literature review. Food Nutr Res 2012;56:19731.

-3 Bath SC, Steer CD, Golding J, Emmett P, Rayman MP: Effect of inadequate iodine status in UK pregnant women on cognitive outcomes in their children: results from the Avon Longitudinal Study of Parents and Children (ALSPAC). Lancet 2013;382:331-337.

4 Hynes KL, Otahal P, Hay I, Burgess JR: Mild iodine deficiency during pregnancy is associated with reduced educational outcomes in the offspring: 9-year follow-up of the gestational iodine cohort. J Clin Endocrinol Metab 2013;98:1954-1962.

5 Vejbjerg P, Knudsen N, Perrild H, Laurberg P, Andersen S, Rasmussen LB, Ovesen L, Jorgensen T: Estimation of iodine intake from various urinary iodine measurements in population studies. Thyroid 2009;19:1281-1286.

-6 Laurberg P, Andersen S, Bjarnadottir RI, Carle A, Hreidarsson A, Knudsen N, Ovesen L, Pedersen I, Rasmussen L: Evaluating iodine deficiency in pregnant women and young infants - complex physiology with a risk of mis- interpretation. Public Health Nutr 2007;10: 1547-1552.

7 Andersen SL, Sorensen LK, Krejbjerg A, Moller M, Laurberg P: Iodine deficiency in Danish pregnant women. Dan Med J 2013; 60:A4657.

8 Gowachirapant S, Winichagoon P, Wyss L, Tong B, Baumgartner J, Melse-Boonstra A, Zimmermann MB: Urinary iodine concentrations indicate iodine deficiency in pregnant Thai women but iodine sufficiency in their school-aged children. J Nutr 2009;139:11691172.

-9 Laurberg P: Thyroxine and 3,5,3'-triiodothyronine content of thyroglobulin in thyroid needle aspirates in hyperthyroidism and hypothyroidism. J Clin Endocrinol Metab 1987; 64:969-974.

10 Rasmussen LB, Jorgensen T, Perrild H, Knudsen N, Krejbjerg A, Laurberg P, Pedersen IB, Bjergved L, Ovesen L: Mandatory iodine fortification of bread and salt increases iodine excretion in adults in Denmark - a 11-year follow-up study. Clin Nutr 2013, Epub ahead of print.

11 Pedersen KM, Borlum KG, Knudsen PR, Hansen ES, Johannesen PL, Laurberg P: Urinary iodine excretion is low and serum thyroglobulin high in pregnant women in parts of
Denmark. Acta Obstet Gynecol Scand 1988; 67:413-416.

12 Kesteloot H, Joossens JV: On the determinants of the creatinine clearance: a population study. J Hum Hypertens 1996;10:245-249.

13 Remer T, Neubert A, Maser-Gluth C: Anthropometry-based reference values for 24-hour urinary creatinine excretion during growth and their use in endocrine and nutritional research. Am J Clin Nutr 2002;75:561-569.

14 Tinggaard J, Aksglaede L, Sorensen K, Mouritsen A, Wohlfahrt-Veje C, Hagen CP, Mieritz MG, Jorgensen N, Wolthers OD, Heuck C, Petersen JH, Main KM, Juul A: The 2014 Danish references from birth to 20 years for height, weight and body mass index. Acta Paediatr 2014;103:214-224.

15 Zimmermann MB: Iodine deficiency. Endocr Rev 2009;30:376-408.

16 Andersen SL, Nohr SB, Wu CS, Olsen J, Pedersen KM, Laurberg P: Thyroglobulin in smoking mothers and their newborns at delivery suggests autoregulation of placental iodide transport overcoming thiocyanate inhibition. Eur J Endocrinol 2013;168:723-731.

17 Aboul-Khair SA, Crooks J, Turnbull AC, Hytten FE: The physiological changes in thyroid function during pregnancy. Clin Sci 1964;27: 195-207. 
18 Davison JM, Hytten FE: Glomerular filtration during and after pregnancy. J Obstet Gynaecol Br Commonw 1974;81:588-595.

19 Brantsaeter AL, Abel MH, Haugen M, Meltzer HM: Risk of suboptimal iodine intake in pregnant Norwegian women. Nutrients 2013; 5:424-440.

20 Bath SC, Walter A, Taylor A, Wright J, Rayman MP: Iodine deficiency in pregnant women living in the South East of the UK: the influence of diet and nutritional supplements on iodine status. Br J Nutr 2014;111:16221631.

21 Soldin OP: Controversies in urinary iodine determinations. Clin Biochem 2002;35:575-579.

-22 Rasmussen LB, Ovesen L, Christiansen E: Day-to-day and within-day variation in urinary iodine excretion. Eur J Clin Nutr 1999; 53:401-407.
23 Als C, Helbling A, Peter K, Haldimann M, Zimmerli B, Gerber H: Urinary iodine concentration follows a circadian rhythm: a study with 3,023 spot urine samples in adults and children. J Clin Endocrinol Metab 2000;85: 1367-1369.

-24 Jakobsen J, Ovesen L, Fagt S, Pedersen AN: Para-aminobenzoic acid used as a marker for completeness of 24 hour urine: assessment of control limits for a specific HPLC method. Eur J Clin Nutr 1997;51:514-519.

25 Leung AM, Braverman LE, He X, Heeren T, Pearce EN: Breast milk iodine concentrations following acute dietary iodine intake. Thyroid 2012;22:1176-1180.

26 Andersen SL, Moller M, Laurberg P: Iodine concentrations in milk and in urine during breastfeeding are differently affected by maternal fluid intake. Thyroid 2014;24:764-772.

-27 Krejbjerg A, Bjergved L, Pedersen IB, Carle A, Jorgensen T, Perrild H, Ovesen L, Rasmussen L, Knudsen N, Laurberg P: Iodine fortification may influence the age-related change in thyroid volume - a longitudinal populationbased study (DanThyr). Eur J Endocrinol 2014;170:507-517.
8 Ategbo EA, Sankar R, Schultink W, van der Haar F, Pandav CS: An assessment of progress toward universal salt iodization in Rajasthan, India, using iodine nutrition indicators in school-aged children and pregnant women from the same households. Asia Pac J Clin Nutr 2008;17:56-62.

29 Zimmermann MB, Aeberli I, Torresani T, Burgi $\mathrm{H}$ : Increasing the iodine concentration in the Swiss iodized salt program markedly improved iodine status in pregnant women and children: a 5-year prospective national study. Am J Clin Nutr 2005;82:388-392.

30 Andersen S, Karmisholt J, Pedersen KM, Laurberg P: Reliability of studies of iodine intake and recommendations for number of samples in groups and in individuals. Br J Nutr 2008; 99:813-818. 